\title{
Transcriptomic response of maize primary roots to low temperatures at seedling emergence
}

\author{
Mauro Di Fenza ${ }^{1,2}$, Bridget Hogg ${ }^{1}$, Jim Grant ${ }^{3}$, Susanne Barth ${ }^{\text {Corresp. } 2}$ \\ ${ }^{1}$ College of Life Sciences, School of Biology and Environmental Sciences, University College Dublin, Dublin D4, Ireland \\ 2 Crops, Environment \& Land Use Programme, Crops Research Centre Oak Park, Teagasc, Carlow R93 XE12, Ireland \\ 3 Research Operations Group, Statistics and Applied Physics Department, Teagasc, Dublin 15, Ireland \\ Corresponding Author: Susanne Barth \\ Email address: susanne.barth@teagasc.ie
}

Background: Maize (Zea mays) is a $\mathrm{C}_{4}$ tropical cereal and its adaptation to temperate climates can be problematic due to low soil temperatures at early stages of establishment. Methods: Therefore, in the current study we have firstly investigated the physiological response of twelve maize varieties, from a chilling condition adapted gene pool, to suboptimal growth temperature during seedling emergence. To identify transcriptomic markers of cold tolerance, in already adapted maize genotypes, temperature conditions were set below the optimal growth range in both control and low temperature groups. The conditions were as follows; control ( $18{ }^{\circ} \mathrm{C}$ for $16 \mathrm{~h}$ and $12{ }^{\circ} \mathrm{C}$ for $8 \mathrm{~h}$ ) and low temperature $\left(12{ }^{\circ} \mathrm{C}\right.$ for $16 \mathrm{~h}$ and $6{ }^{\circ} \mathrm{C}$ for $8 \mathrm{~h}$ ). Four genotypes were identified from the condition adapted gene pool with significant contrasting chilling tolerance. Results: Picker and PR39B29 were the more cold-tolerant lines and Fergus and Codisco were the less coldtolerant lines. These four varieties were subjected to microarray analysis to identify differentially expressed genes under chilling conditions. Exposure to low temperature during establishment in the maize varieties Picker, PR39B29, Fergus and Codisco, was reflected at the transcriptomic level in the varieties Picker and PR39B29. No significant changes in expression were observed in Fergus and Codisco, following chilling stress. A total number of 64 genes were differentially expressed in the two chilling tolerant varieties. These two varieties exhibited contrasting transcriptomic profiles, in which only four genes overlapped. Discussion: We observed that maize varieties possessing an enhanced root growth ratio under low temperature were more tolerant, which could be an early and inexpensive measure for germplasm screening under controlled conditions. We have identified novel cold inducible genes in an already adapted maize breeding gene pool. This illustrates that further varietal selection for enhanced chilling tolerance is possible in an already preselected gene pool. 
1

2

3

4

5

6 1Teagasc Crops, Environment \& Land Use Programme, Crops Research Centre Oak Park,

7 Carlow, R93 XE12, Ireland

$8{ }^{2}$ College of Life Sciences, School of Biology and Environmental Sciences, University College

9 Dublin, D4, Ireland

10

11

12

13

${ }^{*}$ Corresponding author:

14 Susanne Barth

15 susanne.barth@teagasc.ie

16 phone: $++353(0) 599170290$

17

18

\section{seedling emergence \\ Transcriptomic response of maize primary roots to low temperatures at}

${ }^{3}$ Teagasc Research Operations Group, Statistics and Applied Physics Department, Ashtown, Dublin 15, Ireland Mauro di Fenza ${ }^{1,2}$, Bridget Hogg 2 , Jim Grant ${ }^{3}$, Susanne Barth ${ }^{1 *}$ 


\section{Abstract}

22 Maize (Zea mays) is a $\mathrm{C}_{4}$ tropical cereal and its adaptation to temperate climates can be

23 problematic due to low soil temperatures at early stages of establishment. Therefore, in the

24 current study we have firstly investigated the physiological response of twelve maize varieties,

25 from a chilling condition adapted gene pool, to sub-optimal growth temperature during seedling

emergence. To identify transcriptomic markers of cold tolerance, in already adapted maize genotypes, temperature conditions were set below the optimal growth range in both control and low temperature groups. The conditions were as follows; control $\left(18{ }^{\circ} \mathrm{C}\right.$ for $16 \mathrm{~h}$ and $12{ }^{\circ} \mathrm{C}$ for 8 h) and low temperature $\left(12{ }^{\circ} \mathrm{C}\right.$ for $16 \mathrm{~h}$ and $6{ }^{\circ} \mathrm{C}$ for $\left.8 \mathrm{~h}\right)$. Four genotypes were identified from the condition adapted gene pool with significant contrasting chilling tolerance. Picker and PR39B29 were the more cold-tolerant lines and Fergus and Codisco were the less cold-tolerant lines. These four varieties were subjected to microarray analysis to identify differentially expressed genes under chilling conditions. Exposure to low temperature during establishment in the maize varieties Picker, PR39B29, Fergus and Codisco, was reflected at the transcriptomic level in the varieties Picker and PR39B29. No significant changes in expression were observed in Fergus and Codisco, following chilling stress. A total number of 64 genes were differentially expressed in the two chilling tolerant varieties. These two varieties exhibited contrasting transcriptomic profiles, in which only four genes overlapped. We observed that maize varieties possessing an enhanced root growth ratio under low temperature were more tolerant, which could be an early and inexpensive measure for germplasm screening under controlled conditions. 
41 We have identified novel cold inducible genes in an already adapted maize breeding gene pool.

42 This illustrates that further varietal selection for enhanced chilling tolerance is possible in an

43 already preselected gene pool.

\section{Introduction}

46 Worldwide, maize is the foremost cereal product and Europe is the third largest producer of 47 maize grain after the United States and China (www.fao.org). However, along with water 48 availability, low temperatures represent one of the major impediments for plant productivity and 49 geographical distribution in the world.

50 The most widespread germplasms of maize around the globe are Dent types, commonly grown in

51 North America, and Flint types, widely grown in Asia, Europe and in Central and South America

52 (Reimer, 2008). The difference between the two types lies in the type of kernel, which is harder

53 in the outer layer of flint corn. Besides, flint germplasm has a very low water content, which

54 makes it better suitable to chilling conditions (Revilla, 2016). Dent corn grows better in warmer

55 climates and it has a high yield potential when grown near optimal temperature conditions.

56 Maize is a $\mathrm{C}_{4}$ tropical plant whose growth range of temperature extends up to $30^{\circ} \mathrm{C}-35^{\circ} \mathrm{C}$

57 (Presterl et al., 2007) and is significantly sensitive to low temperature, particularly in the early

58 growth stages. Nevertheless, hybrids derived from highland maize can adapt to lower

59 temperatures than the optimal range (Greaves, 1996). However, temperatures below the optimum

60 cause a steady decline of growth of maize, which stops around $6-8^{\circ} \mathrm{C}$. Prolonged exposure to low

61 temperatures involves irreversible cellular and tissue injury (Greaves, 1996), and the effect is 
62 mainly marked in the early growth stages as it impairs several developmental and physiological

63 processes (Marocco, Lorenzoni \& Fracheboud 2005).

64 Chilling is responsible for yield losses and lower metabolisable energy content (starch, sugar) in maize (Frei, 2000); in particular, it affects photosynthesis due to an over excitation of the

66 Photosystem II (PSII) reaction centres and a concomitant production of oxygen radicals (ROS),

67 which are demonstrated to produce injurious effects to the photosynthetic apparatus (Nie, Long 68 \& Barker, 1992).

In Northern and Central Europe maize is generally sown in the last week of April, when soil temperature is warm enough for seeds to germinate, and harvested in autumn before the first air frost occurs damaging the crop with temperatures below $-2^{\circ} \mathrm{C}$. The date of sowing and the date

72 of harvest determine the length of the growing season and therefore the level of maturity and quality of the crop. Early maturing varieties reach maturity earlier; this means that the development of the canopy occurs earlier and so does its closure reducing, this way, the risk of yield losses that can be caused by the first autumn air frost at the end of the growing season. However, despite the improvement in crop quality and yield, these cultivars are still dependent on suitable soil temperatures for the initial establishment of the seedlings and they still benefit from a longer growing season.

Maize root growth generally occurs between $9^{\circ} \mathrm{C}$ and $40^{\circ} \mathrm{C}$, but it has been demonstrated that, 80 during the early phases of development, maize growth is dependent on soil temperature which ranges from $10^{\circ} \mathrm{C}$ to $17^{\circ} \mathrm{C}$ according to the trait and variety (Blacklow, 1972). As the

82 temperature decreases, morphological changes occur in the root system; roots become swollen behind the tip and thicker, with a higher number of seminal roots (Farooq et al, 2009). The effect 
84 of low temperature on roots may be indirectly reflected on shoot elongation and leaf formation 85 (Hund et al., 2007).

86 The use of biodegradable polythene films distributed on the soil surface has helped solve the soil

87 temperature issue in practical crop husbandry giving maize growth a significant enhancement 88 (Keane, 2002), but further varietal improvements through breeding are required to make the 89 maize crop more economical in Northern and maritime Europe.

90 A better understanding of the developmental stages that are particularly sensitive to low 91 temperature will help improve maize adaptation to temperate climates. Each physiological and 92 biological process can be more or less susceptible to suboptimal temperatures, depending on 93 what is called thermal threshold, which is the sub optimal temperature at which the maize hybrid 94 is able to maintain high rates of growth. The lower the thermal threshold the higher is the growth 95 rate and the faster is emergence from soil under low temperatures (Greaves, 1996). The thermal 96 threshold is controlled by specific genes which regulate specific processes at specific 97 developmental stages. The combined processes with lower thermal thresholds will result, 98 therefore, in an optimised growth under low temperature conditions.

99 Plant breeding is still dependant on phenotypic selection, where new hybrids are tested in yield 100 trials and, therefore, selected on the harvestable yield rather than on their ability to cope with 101 chilling temperatures. Chilling tolerance is controlled by genes that are not directly involved in 102 yield, but they contribute to it by conferring tolerance and thus aiding the plant to reach its full 103 yield potential (Greaves, 1996). 
104 The detection of the transcripts and the identification of the genes associated to them will lead, 105 with an appropriate breeding programme, to the transfer of the traits of interest to new hybrids 106 with an improved tolerance to low temperatures. Gene expression profiling can be viable with 107 the employment of technologies like microarray and qPCR capable to screen a large set of 108 transcripts or even the entire transcriptome.

109 In this study, we have investigated the physiological response of primary roots to low 110 temperatures at early seedling emergence in twelve commercially available chilling tolerant 111 maize cultivars differing in kernel type and maturing time. Two pairs of varieties, one with the 112 highest and the other with the lowest growth response were selected for gene expression 113 profiling. As the genotypes were known to be cold tolerant and in order to identify specific genes

114 that are regulated in low temperatures conditions such as in maritime temperate climates, both 115 control and stress climate conditions were set to sub-optimal temperatures such as typical growth 116 conditions in Ireland. This was aimed at the identification of novel transcripts conferring 117 enhanced chilling tolerance in an already adapted maize breeding gene pool.

Material and Methods

Plant material and growth conditions

121 Untreated maize seeds for the physiological experiment were provided by the seed companies 122 Caussade (France), Pioneer (France) and Codisem (France), for a total of twelve varieties 123 (Supplemental Materials Table 1). Varieties Algans, Justina and Picker were included in the Irish 124 Recommended List 2008 of the cultivars that have shown a high yield performance under Irish 
125 climate conditions in trials. The experimental varieties also differed in the type of kernel (Flint, 126 Dent, and Flint-Dent) and maturity type.

127 Two independent non seed coating treated 45-seed groups of each variety were germinated in 128 growth chambers (Snijder Microclima 1750, The Netherlands) under control and low 129 temperature conditions on a surface of capillary matting lying over two layers of blotting paper 130 soaked with $100 \mathrm{ml}$ of distilled water. The blotting paper and capillary matting were placed in $13152 \mathrm{~cm} \times 42 \mathrm{~cm} \times 9 \mathrm{~cm}$ seed trays, which were covered by another inverted seed tray to reduce 132 water loss. The blotting paper was placed below the single layer of capillary matting. Each group 133 of 45 seeds, were arranged as two sub-groups of 21 and 24 seeds in two separate seed trays. 134 Seeds were used directly from the seed bag and placed under two controlled growth conditions. 135 The control temperature regime was set at $18^{\circ} \mathrm{C}$ for 16 hours and $12^{\circ} \mathrm{C}$ for 8 hours; the low 136 temperature regime was set at $12^{\circ} \mathrm{C}$ for 16 hours and $6^{\circ} \mathrm{C}$ for 8 hours. Control temperature 137 conditions were chosen like at an ideal spring day when maize is being sown in temperate 138 climates like in Ireland. This allowed us to target the identification of transcripts which are up- or 139 down regulated in germplasm which has been bred to perform in the maritime climate of the 140 northern hemisphere. The experiment was conducted in constant dark conditions with $40 \%$ of 141 relative humidity.

Germination assessment and growth ratio $(G R)$

144 Seed germination was classified as such when the radicle emerged from the meristem and was at 145 least $1 \mathrm{~mm}$ long. Shoot measurements were taken as an indirect assessment of root growth 146 performance. Seed germination was recorded in the number of days from sowing to radicle 
147 emergence (seed germination data under control and cold stress treatments in Supplemental

148 Materials Table 1). The length of the primary roots and the shoots were measured on germinated

149 seeds with similar root length under the same growth conditions at 24-hour intervals over a

150 period of five days post-germination (time points). Trays were daily watered with $100 \mathrm{ml}$ of

151 distilled water. The response to low temperature was calculated at every time point and was

152 expressed as growth ratio (GR). The GR was calculated dividing the average length of roots and 153 shoots of each variety at low temperature by the average length of root and shoot of each variety 154 at control temperature. GR was expressed in percentage.

Analysis of the physiological response

157 The experiment was a randomised block design and was conducted as three independent 158 experiments. A three-way ANOVA was fitted including a blocking factor (experiment) and three 159 effects (genotype, treatment and time point) with all the possible interactions of interest 160 (genotype, treatment, time point, genotype $x$ treatment, genotype $x$ time point and genotype $x$ 161 treatment $x$ time point). The measurements over time were made on the same experimental units 162 and a repeated measures analysis incorporating a covariance structure was used to model this 163 lack of independence. P-values $<0.05$ were taken as significant. Residual checks were made to 164 ensure that the assumptions of the analysis were met and responses were log transformed to 165 correct skew and/or non-constant variance as appropriate. The analysis was performed with the 166 GenStat statistical software package (VSN International, UK) and the Statistical Analysis System 167 (SAS) software (SAS Institute Inc., USA). 
170 Total RNA was isolated of three independent biological replicates from $3 \mathrm{~cm}$ maize roots, on 171 days 1, 2, 3, 4, 5 post-germination. Roots were excised and snap frozen in liquid nitrogen and 172 stored at $-80^{\circ} \mathrm{C}$. Frozen root samples were homogenised in $1.5 \mathrm{ml}$ microcentrifuge tubes with the 173 use of tube pestles (Sigma-Aldrich $\AA$, Germany) and used as a starting material for RNA 174 extraction. The isolation of total RNA was carried out with the RNeasyC Plant Mini Kit 175 (Qiagen, UK). Samples were treated with DNase I from the RNase-free DNase set (Qiagen, UK). 176 Quantity and quality of RNA were determined with an Agilent 2100 BioAnalyzer (Agilent 177 Technologies, USA). Only RNA samples with an RNA integrity number (RIN) $\geq 8$ assigned by 178 the BioAnalyzer were used for microarray analysis.

Three independent root tissue RNA samples on day 1 post-germination (time point 1) were used

182 for the microarray experiment, for a total of 24 samples. One $\mu \mathrm{g}$ of each RNA sample was 183 hybridised on a 46K 70-mer oligo array developed in the 'maize oligonucleotide array project' by the University of Arizona, The Institute for Genomic Research (TIGR) and the University of Wisconsin (Seifert et al., 2012). The 46k array was configured as 4 rows and 12 columns. The intersection of a row with a column represented a subarray. Each subarray consisted in 31 columns and 31 rows. A two-colour microarray was used to compare each variety from the control and the same variety from low temperature according to a loop design. The array hybridisation with $\mathrm{Cy} 3$ and $\mathrm{Cy} 5$ dyes was conducted by the Institute of Genomic Research in 
191 to manufacturer's instructions. Image acquisition was conducted with a GenePix ${ }^{\circledR}$ scanner

192 (Axon, USA) as a service in Arizona.

193 The analysis of microarray was performed with CARMAweb (Comprehensive R-based

194 Microarray Analysis web service, https://carmaweb.genome.tugraz.at/carma/) a web application

195 based on the R (http://www.r-project.org/) programming language and environment for statistical 196 computing. CARMAweb implements the Bioconductor limma (Linear Model for Microarray

197 Data) package for R, specifically designed for microarray analysis. Data were quality checked, 198 adjusted and normalised before analysis to remove the technical variance and systematic errors 199 without altering the biological variance within the data.

200 The data were $\log _{2}$ transformed, background corrected and normalised. Background optical noise 201 of the hybridisation was corrected with the minimum method, which consists in giving the half 202 the minimum positive corrected intensities for the array to any intensity that is equal to zero or 203 negative. Within-array normalisation was performed with the print-tip loess method and 204 between-array normalisation was carried out by scaling the expression values.

205 The statistical analysis was restricted to the $40 \%$ of the probe sets in order to reduce the loss of 206 power of the test. When many thousands of simultaneous hypothesis tests are performed, the 207 probability of type I errors grows with the number of tests and the power becomes critical. So, a 208 pre-filtering of the data was applied to remove from consideration the set of genes that is not 209 differentially expressed under any comparison and thus to run the analysis on the $40 \%$ of the 210 genes with the biggest variance. Differentially expressed genes were determined by subtracting 211 the average expression of the gene in the control from the average expression of the gene under 212 treatment. Bad array spots were excluded from the analysis. Adjusted p-values were generated 
213 using the Benjamini and Hochberg method to correct for multiple testing in the experiment

214 (Benjamini \& Hochberg, 1995). The analysis was performed using the limma paired moderated

215 t-test statistics, based on the empirical Bayesian approach.

216 Microarray data were deposited to GEO (Gene Expression Omnibus) under accession number 217 GSE72508.

219 Real Time qPCR

220

221

222

223

224

225

226

Microarray data validation was performed with three independent replicates on day 1 postgermination. Reverse transcription was conducted with 500 ng total RNA, $1 \mu 110 \mathrm{mM}$ dNTPs (Bioline, UK), $1 \mu$ l oligo-dT(20) primers (Invitrogen, UK) and incubated at $65^{\circ} \mathrm{C}$ for $5 \mathrm{~min}$ in a volume of $11 \mu 1$. After incubation the solution was chilled on ice for $1 \mathrm{~min}$ and $4 \mu \mathrm{l}$ of $5 \mathrm{X}$ FS reaction buffer, $1 \mu 10.1 \mathrm{M}$ DTT, $1 \mu 1$ RNase free H2O (Qiagen, UK) and $1 \mu 1$ SuperScript ${ }^{\circledR}$ III reverse transcriptase (Invitrogen, UK) were added bringing the volume to $20 \mu 1$. The reaction was incubated at $50^{\circ} \mathrm{C}$ for $60 \mathrm{~min}$ and inactivated at $70^{\circ} \mathrm{C}$ for $15 \mathrm{~min}$. The solution was brought up to a final volume of $50 \mu \mathrm{l}$ by adding RNase free $\mathrm{H}_{2} \mathrm{O}$.

Relative Real Time PCR was conducted with an ABI 7500/7500 Fast Real Time PCR system (Applied Biosystems, USA) using the Fast Sybr ${ }^{\circledR}$ Green Master Mix (Applied Biosystems, USA) according to the manufacturer's instructions, but in a reaction volume of $10 \mu 1$.

All primers were designed using the Primer3Plus, an advanced Prime3 designer tool (Untergasser et al., 2007). Lyophilised primers were re-suspended in nuclease free water to a final concentration of $100 \mathrm{pmol} \mu \mathrm{l}^{-1}(\mathrm{mM} / \mathrm{L})$. Re-suspended primers were diluted to the working 
234 solution of $10 \mathrm{pmol}^{-1}$. A five series dilution standard curve was used to test primer efficiency.

235 As the efficiency of all the primers was $\geq 95 \%$, the Livak's method ( $\Delta \Delta \mathrm{Ct}$ method) was used to 236 calculate the relative expression. Four genes (Supplemental Materials Table 3) were tested as

237 housekeeping genes with the geNorm algorithm (Vandesompele et al., 2002). Adh and Ef1- $\alpha$ 238 were finally used as housekeeping genes. The target genes used for Real Time qPCR were 239 selected out of the top 100 up- or down regulated genes with the smallest p-values (primer 240 sequences of all candidate genes and housekeeping genes in Supplemental Materials Table 4).

241 Three independent replicates on day 1, 2, 3, 4 and 5 post-germination were used to investigate 242 the difference in gene expression of the target genes across five time points in a time series 243 experiment. Fold-change was calculated at every time point by subtracting the average 244 expression of the gene in the control from the average expression of the gene under treatment.

245 The relative expression across time points was calculated by subtracting the fold-change on day 2461 post-germination from the fold-change of the other days post-germination.

\section{Results}

Physiology and genetics of maize roots grown at low temperatures

250 The physiological response of maize to low temperature was analysed in twelve maize varieties

251 differing in kernel type and maturity group (Supplemental Materials Table 1). Overall all of

252 these twelve varieties still had root and shoot growth under chilling treatment and thus all of 253 them could be broadly considered as chilling tolerant (Figure 1). However for root and shoot 254 growth, there was a significant variety $x$ treatment $x$ time point interaction (Table 1) resulting in 
255 an effect of variety on treatment and time point at which the measurements were taken. For this

256 reason the physiological response was calculated as a chilling stress/control ratio (see Materials

257 and Methods) and not simply the root and shoot growth under chilling stress. The twelve

258 varieties exhibited a root response pattern different to the shoot pattern. However, the shoot

259 growth was measured as in indirect effect of low temperature on root elongation, so, to

260 determine the most cold tolerant and the most cold sensitive genotypes the Tukey's range test (a

261 multiple comparison method) was performed on the root growth ratio (Figure 1). The

262 physiological response to low temperatures has led to the identification of two groups of

263 genotypes with contrasting cold tolerance. The varieties Picker and PR39B29 showed the highest

264 resistance to chilling stress in terms of both root and shoot growth, while Algans and Justina

265 presented the lowest degree of tolerance. However, because of their poor ability to germinate

266 under the cold stress temperature regime, Algans and Justina were excluded for subsequent

267 microarray analysis and substituted by the second most sensitive pair of varieties, Codisco and

268 Fergus. Therefore, the four final genotypes used for the gene expression profiling were Picker,

269 PR39B29, Codisco and Fergus.

271 Microarray analysis

272 The microarray analysis of root tissue showed that the most chilling tolerant varieties Picker and

273 PR39B29 have, each, a set of differentially expressed genes (up and down regulated, p-value

$274<0.05)$, while no genes were listed for the other two less chilling tolerant varieties Fergus and

275 Codisco, indicating that no significant change in expression was found in any of the genes

276 analysed (Table 2). The overall number of genes up and down regulated in the two more chilling 
277 tolerant varieties amounted to 64 (Supplemental Figure 1), which are, however, divided in a 278 group of 39 genes in PR39B29 (Supplemental Materials Table 5) and 30 genes in Picker

279 (Supplemental Materials Table 6), as the two varieties exhibited two different transcriptomic 280 patterns in which only four genes were shared, although not all with the same degree of 281 regulation (Supplemental Materials Tables 5 and 6). These four genes were a RNA binding 282 protein (MZ00003507), a pathogenesis related protein-1 - maize (MZ00004486), a hypothetical 283 protein (MZ00022876) and an unknown protein (MZ00041708). The Gene Ontology (GO) 284 functions of the genes were available in the probe dataset. However, no information for 285 MZ00003507 was available and the nearest match obtained for this sequence was an RNA binding protein in Arabidopsis thaliana. Microarray data of our hybridization experiments were deposited to GEO (Gene Expression Omnibus) under accession number GSE72508.

$q R T-P C R$ for data validation

290

291

292

293

294 295

296

297

298

Nine differentially expressed genes, including the four in common, were selected to validate the microarray analysis with qRT-PCR. Real Time PCR was performed on three independent biological replicates. The $\log _{2}$ average expression values of the qRT-PCR at time point 1 were correlated to the $\log _{2}$ values (M-values) of the microarray analysis for validation. Spearman's correlation (rho) for non-parametric distribution was derived from the square root of $\mathrm{R}^{2}$, which was $88 \%$. This strong correlation between the two expression profiling techniques assessed that microarray data were successfully validated by qRT-PCR.

qRT-PCR was also performed to investigate the expression pattern of the genes of interest in Picker and PR39B29 across five time points corresponding to number of days post germination. 
299 Gene expression was not maintained over the time (Figure 2), but was subjected to fluctuation.

300 Except for RBP, the gene expression pattern was similar between the two varieties, in particular 301 for PRP-1.

\section{Discussion}

Investigation on the cold tolerance in maize has mainly focused on the early phases of growth, as it is known that plant establishment is fundamental for the crop to reach maturity and maximum development. The photosynthetic apparatus is susceptible to low temperatures (Stamp, 1984), therefore most of the attention has been focused on the effect of sub-optimal temperatures on photosynthesis during leaf development. When maize is grown at sub-optimal temperatures, impaired chloroplast function through photo inhibition as well as altered pigment composition and chlorophyll deficiency result in a damaged photosynthetic apparatus (Greaves, 1996; Marocco, Lorenzoni \& Fracheboud, 2005). Besides the photosynthetic performance of seedlings is influenced by the different cold tolerance in root and shoot development (Stamp, 1984; Tollenaar \& Lee, 2002). Germination and the heterotrophic phase of development are also considerably impaired when soil temperatures are too low for a functional root system to establish.

Studies on roots are generally performed on field grown maize, although this approach is not free

317 from flaws, mostly due to the destruction of root material during sampling. The analysis is usually done by image acquisition, which can be biased because of the background noise due to soil (Dong et al., 2003). Over the years, hydroponic cultivation (Sanguineti et al., 1998) or the use of sand columns (Ruta et al., 2010) have been adopted to grow roots under controlled 
321 experimental environments, although there are sampling limitations. The acquisition of data on

322 root traits can be carried out in several ways, from recording roots using a simple camera or

323 photocopier (Liedgens \& Riechner, 2001; Collins et al., 1987), a scanner (Dong et al., 2003;

324 Hund, Trachsel \& Stamp, 2009) to X-ray imaging techniques (Gregory et al., 2003). Root image

325 analysis has progressed to the development of advanced software for the quantitative analysis or

326 root growth and the architecture of complex root systems (Lobet, Pages \& Draye, 2011).

327 However, these techniques can be invasive and damage the root samples as well as reduce the

328 sample size under consideration. Besides, growth in the field is significantly influenced by

329 several environmental cues, which makes cold tolerance difficult to sunder from other stresses

330 (Riva Roveda, 2016). It is therefore necessary to implement an adequate controlled growth

331 environment, use techniques that are not destructive and take repetitive measurements of the

332 traits of interest, giving temporal information about root growth over a certain period of time.

333 Climate chambers provide appropriate and reproducible conditions to assess cold tolerance and

334 predict the growth potential of maize seedlings by physiological characteristics (Stingens et al.,

335 2013; Stamp, 1986). The growth of maize seedlings on blotting paper for root development

336 analysis used in this work has been outlined previously, with the specific aim of developing a

337 phenotyping platform for non-destructive and repeated measurements of root growth (Hund, et

338 al., 2009).

339 To date, analysis of gene expression in chilling stressed maize roots has not been carried out.

340 Maize genotypes can be distinguished as cold tolerant and cold sensitive varieties: dent hybrids

341 shows a cold sensitive phenotype, whilst flint hybrids are cold tolerant (Revilla et al., 2016).

342 Whereas low temperatures seriously injure the cold sensitive varieties, the cold tolerant

343 genotypes adjust their metabolism to adapt to the environmental conditions. They grow through 
344 the activation of metabolic mechanisms that increase the content of specific molecules, such as

345 cryo-protective compounds and antioxidants, but they also involve the down-regulation of some

346 other gene products such as acquaporins (Janska et al., 2010).

347 Root and shoot growth was carried out in controlled environmental chambers in a range of

348 temperature regimes that are in accordance with previous studies (Blacklow, 1972; Farooq et al.,

349 2009; Marocco et al., 2005). Germination requires a minimum temperature of approximately

$35010^{\circ} \mathrm{C}$ (Levitt, 1980) and cellular and tissue damage can occur when temperature is below $5^{\circ} \mathrm{C}$

351 (Greaves, 1996). Prolonged exposure below this temperature can seriously injure seedlings that

352 are no longer able to recover (Theocharis, Clement \& Barka, 2012). Although, the environmental

353 conditions applied in our study do not cause deleterious effect on the maize seedlings, they both

354 are sub-optimal chilling temperatures, as chilling is defined as a temperature range of $5-15^{\circ} \mathrm{C}$

355 (Nguyen et al, 2009). More specifically, the control temperature applied in this study is defined

356 as a mild-chilling stress compared to the other stress that is defined as strong-chilling (Marocco

357 et al., 2005). The reason behind this choice is duplex: firstly, we wanted to represent an ideal

358 spring day when maize is being sown in temperate climates like Ireland. This allowed us to

359 target transcripts that are differentially regulated in the germplasm, which has been bred to

360 perform in the maritime climate of the Northern hemisphere; secondly, as the cultivar were cold

361 tolerant, we believed that comparing the gene expression under cold stress with the gene

362 expression under optimal growth conditions would hide the genetic difference among the

363 varieties and would outline a general common response to low temperature. In support of our

364 hypothesis, a recent study has showed that even genotypes with contrasting cold sensitivity

365 regulate thousands of common genes that are probably involved in a general response to cold

366 stress, whilst only a few are responsible for the genetic difference between the germplasms 
367 (Sobkowiak et al., 2014). Therefore, by applying more stringent control environmental 368 conditions, we narrowed down our scope to those few genes that could play a dominant role in 369 cold tolerance and that could highlight the genetic difference not only between cold tolerant and 370 cold sensitive cultivars, but also within cold tolerant genotypes. In our study, cold tolerance was 371 assessed phenotypically, on the results of the physiological experiment, which outlined two 372 contrasting groups, of which a pair of varieties each was destined to gene expression profiling. A 373 pair of varities each group allowed us to detect genetic difference between and within the groups 374 and potential different strategies used to cope with cold stress. The microarray analysis outlined 375 a set of differentially expressed genes whose number (64) is consistent with the number of differntially expressed genes in Sobkowiak's work (66) and five genes (MZ00004711, MZ00018470, MZ00026737, MZ00041500, MZ00043117) are outlined in both studies, although three (MZ00004711, MZ00026737, MZ00041500) were only regulated in Picker and two (MZ00018470, MZ00043117) only in PR39B29. Interestingly, the microarray analysis has revealed that the transcriptome was differently regulated only in two of the four varieties (Picker and PR39B29), precisely, the varieties whose root growth ratio was significantly higher, thus supporting the evidence that the regulation of specific genes confers higher cold tolerance (Theocharis, Clement \& Barka, 2012). As previously stated, these results should not be unexpected as the growth temperatures used for this study were sub-optimal, therefore, common cold regulated genes like those controlling the inducer of cbf expression (ICE)-C repeat binding factor/DRE binding factor1 (CBF/DREB1) transcriptional pathway (Miura \& Furumoto, 2013) could have been triggered in both control and stress conditions and, therefore, no difference in expression was detected by microarray for those genes. However, transcriptomic differences were still observed in Picker and PR39B29. Codisco and Fergus were probably non able to 
390 trigger a specific response to cope with cold stress and therefore, no difference in expression was

391 detected for these varieties.

392 Although signicant $(\mathrm{P}<0.05)$ contrasting cold tolerance was observed between PR39B29 (flint)

393 and Fergus (dent) as the kernel type would suggest, stastical analysis showed that, in our

394 experiment, neither kernel or maturing time were sufficient conditions for conferring better

395 growth performance (Supplementary Materials Table 2). Cultivars with the same maturing time

396 or the same kernel exhibited opposite adaptation to low temperatures, or even both cold tolerant

397 and cold sensitive phenotypes (Huski, PR39D60; P <0.05). However, when showing both mid-

398 early and flint-dent phenotypes (Crazi, Clariti, Algans and Codisco), the cultivars showed no

399 statistical difference among them $(\mathrm{P}<0.05)$ in root growth. In spite of this, Crazi and Clariti also

400 exhibited both cold tolerant and cold sensitive genotypes $(\mathrm{P}<0.05)$, therefore making them

401 unsuitable for assessing cold tolerance. Nevertheless, the number of replicates for each

402 individual trait is too small to assess cold tolerance on a phenotypical basis (Fergus is the only

403 dent variety).

404 Gene Ontology showed that the differentially expressed genes in Picker and PR39B29 are mostly 405 involved in molecular functions and biological processes, some of which, such as peroxidases, 406 patogenesis related proteins, RNA binding factors and the plant specific NAC transcription 407 factor, have been demonstrated to be induced in response to cold stress (Nie et al., 1992; Edreva, 408 2005; Lorkovic, 2009; Puranik et al., 2012).

409 Interestingly, the microarray analysis outlined two different profiles for Picker and PR39B29, 410 suggesting that, even though the two genoytpes had the same $(\mathrm{P}<0.05)$ physiological response to 411 cold stress, they might trigger different biological pathways to cope with low temperatures. This 
412 is the case of an increase in pepditases and proteinases that was observed only in PR39B29.

413 Proteolytical enzymes have been shown to be indispensible in maintaining the physiological state

414 of the plant cells by degrading potentially harmful and irreversibly damaged proteins in response

415 to draught stress (Vaseva et al., 2012). Draught stress is strongly associated with cold stress as

416 chilling is responsible for reduced root hydraulic conductance (Aroca et al., 2003). This explains

417 why the NOD protein was also induced. NOD23-like membrane integral proteins belong to a

418 sub-family of root specific acquaporins, which mediate cold acclimation in plants by regulating

419 root hydraulic conductivity (Ahamed et al., 2012). In PR39B29 cold stress also induced the

420 plant-specific $N A C$ protein, which belongs to a major transcription factor family that has been

421 previously demonstrated to be responsible in the adaptation of plants to environmental stress

422 (Puranik et al., 2012).

423 Picker showed, as opposed to PR39B29, repression of most of the differentially expressed genes, 424 in particular of metabolic enzymes, ribosomal proteins (rpl2, rpl16) and even of an isoform of 425 peroxidases, which are widely known to act against oxidative stress induced by environmental 426 cues (Kocsy et al., 2001). The lpr2 gene has been shown to be down regulated in soybean, where 427 the arrest of protein synthesis represents a strategy that plants use to cope with stress to promote 428 a quick adaptation in stressful environments (Ludwig \& Tenhaken, 2001). Maize plants can even 429 enter a stand-by mode to adapt and quickly recover after a mild-chilling stress (Riva-Roveda et 430 al., 2016).

431 The analysis of the trascriptomes of Picker and PR39B29 has demonstrated that specific genes 432 need to be induced, while others need to be repressed. However, the strategy used by both the 433 cultivars required depletion of proteins and interruption of their synthesis. 
434 Interestingly, four genes (MZ00003507, MZ00004486, MZ00022876 and MZ00041708) were 435 differentially expressed in both Picker and PR39B29. These genes could be representative of a common response used by cold tolerant varieties to cope with low temperatures. The first of these four common genes encodes for the pathogenesis related protein 1 (PR-1). PR-1 belongs to a group of PR-families that are induced in response to several abiotic stressful environmental stimuli, including wounding and low temperatures (Van Loon, Rep \& Pieterse, 2006). The second shared gene encodes for a putative RNA binding protein (RBP). RBPs are known to be involved in the post-transcriptional regulation of RNAs, modulating gene expression during 442 development and in the adaptation of plants in response to environmental stresses (Lorkovic, 2009). However, the expression of this gene is contrasting in the two cold tolerant genotypes: it

444 is induced in Picker as opposed to the majory of the genes being down regulated, and it is repressed in Picker, where most of the genes are up regulated. This contradictory regulation makes its exact role in cold tolerance unclear. The other two genes (MZ000022876 and 447 MZ00041708) encode for hypotethical proteins whose function is unknown and protein family analysis (http://pfam.xfam.org/) did not reveal a match for their protein sequences that could 449 have helped understand their role in cold tolerance. These genes may represent novel cold induced transcripts in an already adapted maize breeding gene pool and should be the basis for more extensive research on transcripts involved in root tissue cold tolerance.

452 As RPB showed opposite levels of expression between Picker and PR39B29, we have 453 hypothisised that gene regulation might not be maintained over time. The different gene 454 expression profiles between the cold tolerant varieties could not be reflecting two strategies for 455 cold acclimation, but two different stages of a unique strategy. After all, the suspension of the synthetic apparatus previously described in soybean and the growth arrest seen in maize are only 
457 transient. Microarray is a useful high troughput screening, but it only captures a photograph of

458 the transcriptome in a specific physiological instant, which is represented by the time point

459 selected. In order to understand whether the difference in gene expression was maintained over

460 time we have investigated the fold change of the four shared genes across five time points. The

461 transcriptomic pattern of these genes showed that the expression was not maintained over time,

462 but it fluctuated. Interestingly, the expression of RBP in PR39B29 went up, while for Picker

463 went down. Even after this analysis the role of RBP remains to be established. As for the other

464 genes, they showed a similar expression pattern between the varieties, suggesting a less variable

465 way to respond to cold stress.

466

467 Acknowledgements

468 We are thankful to seed companies supplying us with non-coated experimental seed. We are

469 indebted to Thomas F. Gallagher, now retired from University College Dublin, who was the 470 project coordinator of this study and the PhD study supervisor of MF. 


\section{Figures}

472

473

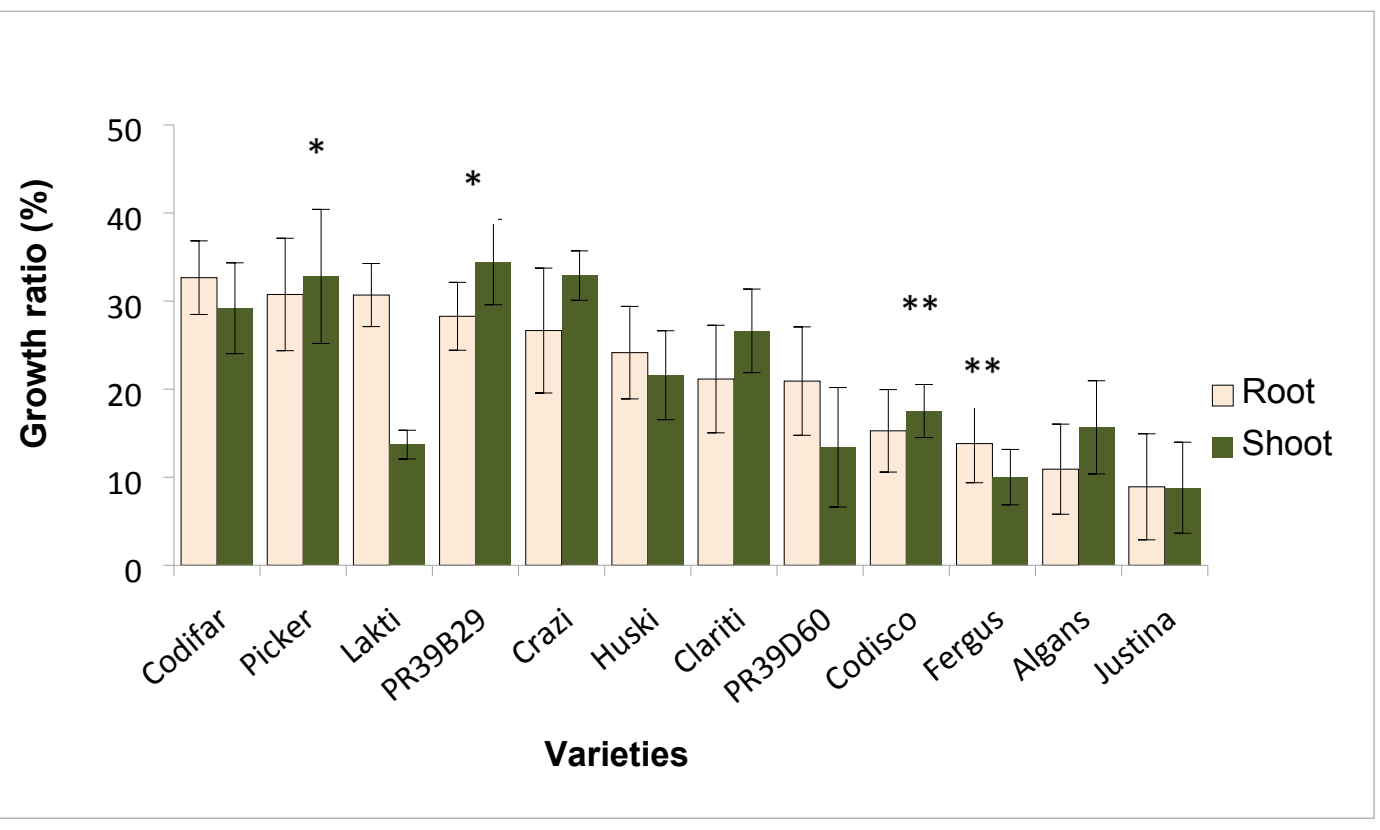

474 Figure 1. Growth ratio as response to cold stress. The growth ratio for roots and shoots was obtained by relating the average length of a root and shoot of cold treatment to the length of root and shoot of control, respectively. Tukey's range test was carried out to determine the two varieties with the highest combined root and shoot response to cold stress (indicated by *) and 478 lowest combined root and shoot tolerance to cold stress (indicated by **). Note: varieties Algans and Justina were excluded from ranking list due to uneven germination rates (Supplemental Table 1). Vertical error bars represent mean $\pm \mathrm{SE}(\mathrm{n}=135)$. The difference between varieties was significant at $\mathrm{p}<0.05$. 

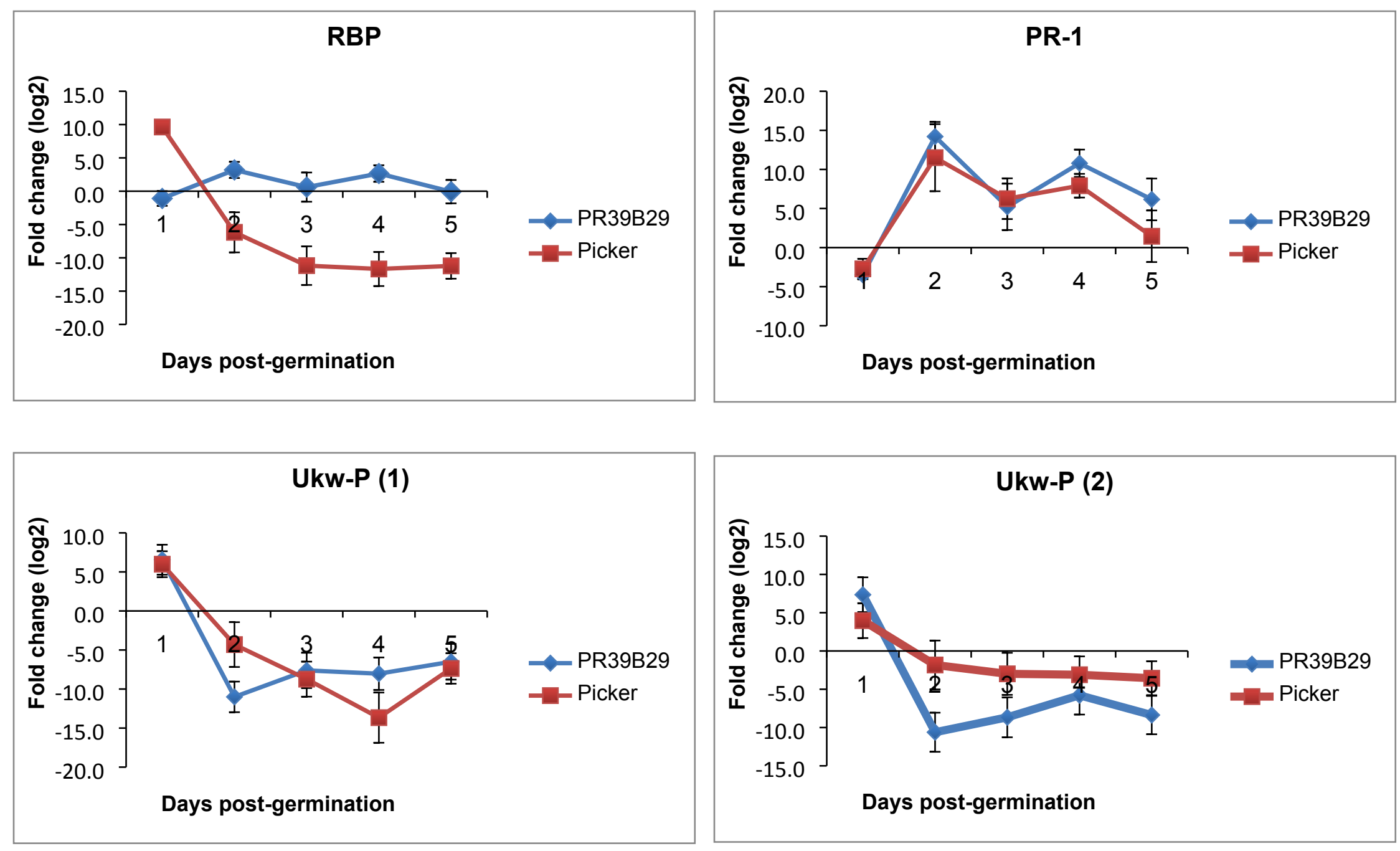
PR39B29. Gene expression patterns were examined over five days post-germination, from day 1 to day 5 . Vertical error bars 
489

Tables

490

491

492 point on growth in twelve maize varieties.

\begin{tabular}{|l|l|l|}
\hline Effect & Root P value & Shoot P value \\
\hline replicate & $<.0001$ & $<.0001$ \\
\hline variety & $<.0001$ & $<.0001$ \\
\hline treatment & $<.0001$ & $<.0001$ \\
\hline variety $x$ treatment & $<.0001$ & $<.0001$ \\
\hline time point & $<.0001$ & $<.0001$ \\
\hline variety $x$ time point & $<.0001$ & $<.0001$ \\
\hline treatment $x$ time point & $<.0001$ & $<.0001$ \\
\hline variety $x$ treatment $x$ time point & $<.0001$ & $<.0001$ \\
\hline
\end{tabular}

493

494

495

496

497

498

499

500

501

502

Table 1. Three-way Analysis of variance (ANOVA) tests of genotype, treatment and time

4

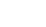

\section{6}




\begin{tabular}{|c|c|c|c|c|c|}
\hline ID & Variety & Mean M & Mean A & Gene product & Abbreviation \\
\hline \multirow[t]{2}{*}{ MZ00003507 } & Picker & 2.43 & 10.59 & RNA binding protein (Arabidopsis thaliana) & RBP \\
\hline & PR39B29 & -2.78 & 10.81 & & \\
\hline \multirow[t]{2}{*}{ MZ00004486 } & Picker & -3.66 & 9.86 & Pathogenesis related protein-1 - maize (Zea mays) & PR-1 \\
\hline & PR39B29 & -2.42 & 9.35 & & \\
\hline \multirow[t]{2}{*}{ MZ00022876 } & Picker & 2.61 & 8.66 & Hypothetical protein (Oryza sativa - japonica cultivar-group) & Ukw-P (1) \\
\hline & PR39B29 & 2.58 & 9.60 & & \\
\hline \multirow[t]{2}{*}{ MZ00041708 } & Picker & 2.18 & 10.16 & Unknown protein (Oryza sativa - japonica cultivar-group) & Ukw-P (2) \\
\hline & PR39B29 & 2.46 & 13.30 & & \\
\hline MZ00023411 & PR39B29 & 2.46 & 11.87 & 22 kDa drought-inducible protein (Saccharum hybrid cultivar) & - \\
\hline MZ00026737 & PR39B29 & 2.55 & 11.62 & Peroxidase (Zea mays) & - \\
\hline MZ00029223 & PR39B29 & -2.47 & 11.38 & $\begin{array}{l}\text { Putative heat shock protein hsp22 precursor (Oryza sativa - } \\
\text { japonica cultivar-group) }\end{array}$ & - \\
\hline MZ00026029 & Picker & -2.17 & 9.82 & Probable lipid transfer protein - rice (Oryza sativa) & - \\
\hline MZ00037140 & Picker & -3.45 & 11.24 & $\begin{array}{l}\text { Glucose starvation-induced protein precursor (clone pZSS2) - } \\
\text { maize (Zea mays) }\end{array}$ & - \\
\hline
\end{tabular}


508

509

510

511

512

513

514

515

516

517

518

519

520

521

522

523

524

525

\section{References}

Ahamed, A., Murai-Hatano, M., Ishikawa-Sakurai, J., Hayashi, H., Kawamura, Y. \& Uemura, M. (2012) Cold stress-induced acclimation in rice is mediated by root-specific aquaporins. Plant \& Cell Physiology. 53, 1445-1456.

Aroca, R., Vernieri, P., Irigoven, J. J., Sancher-Diaz, M., Tognoni, F., \& Pardossi, A. (2003). Involvement of abscisic acid in leaf and root of maize (Zea mays L.) in avoiding chillinginduced water stress. Plant Science. 165, 671-679.

Barlow, P. W. \& Adam, S. J. Anatomical disturbances in primary roots of Zea mays following periods of cool temperature. Environ. Exp. Bot. 29, 323-36 (1989).

Benjamini, Y. \& Hochberg, Y. Controlling the false discovery rate: A practical and powerful approach to multiple testing. J. R. Stat. Soc. Series B Stat. Methodol. 57, 289-300 (1995).

Blacklow, V. M. Influence of temperature on germination and elongation of the radicle and shoot of corn (Zea mays L.). Crop Sci. 12, 647-50 (1972).

Collins, R. P., Gregory, P. J. Rowse, H. R., Morgan, A. \& Lancashire B. Improved methods of estimating root length using a photocopier, a light box and a bar code reader. Plant Soil 103, 277-80 (1987).

Dong, S. F., Neilsen, D., Neilsen, G. H. \& Weis M. A scanner-based root image acquisition technique for measuring roots on a rhizotron window. HortScience 38, 1385-88 (2003). 
526 Edreva, A. Pathogenesis-related proteins: Research progress in the last 15 years. Gen. Appl. $527 \quad$ Plant Physiology 31, 105-124 (2005).

528 Farooq, M., Aziz, T., Wahid, A., Lee, D. J. \& Siddique K. H. M. Chilling tolerance in maize: 529 Agronomic and physiological approaches. Crop Pasture Sci. 60, 501 (2009).

530 Fracheboud, Y. Identification of quantitative trait loci for cold-tolerance of photosynthesis in 531 maize (Zea mays L.). J. Exp. Bot. 53, 1967-77 (2002).

532 Frei, O. M. Changes in yield physiology of corn as a result of breeding in Northern Europe. $533 \quad$ Maydica 45, 173-83 (2000).

534 Greaves, J.A. Improving suboptimal temperature tolerance in maize - the search for variation. $J$. 535 Exp. Bot. 47, 307-23 (1996).

536 Gregory, P. J., Hutchison, D., Read, D. B., Jenneson, P. M. \& Gilboy, W. B. Non-invasive 537 imaging of roots with high resolution X-Ray micro-tomography. Plant Soil 255, 351-59 $538 \quad(2003)$.

Hund, A., Trachsel, S. \& Stamp, P. Growth of axile and lateral roots of maize: I development of 540 a phenotying platform. Plant Soil 325, 335-49 (2009).

Hund, A., Fracheboud, Y., Soldati, A., Frascaroli, E., Salvi, S. \& Stamp, P. QTL controlling root 542 and shoot traits of maize seedlings under cold stress. Theor. Appl. Genet. 109, 618-29 (2004). 
544 Janská, A., Marsík, P., Zelenková, S. \& Ovesná J. Cold stress and acclimation - What is

545 important for metabolic adjustment? Plant Biol. 12, 395-405 (2010).

546 Lobet, G., Pagès, L. \& Draye, X. A novel image-analysis toolbox enabling quantitative analysis 547 of root system architecture. Plant Phys. 157, 29-39 (2011).

548 Lorković, Z. J. Role of plant RNA-binding proteins in development, stress response and genome 549 organization. Trends Plant Sci. 4, 229-36 (2009).

550 Presterl, T., Ouzunova, M., Schmidt, W., Möller, E. M., Röber, F. K., Knaak, C., Ernst, K., 551 Westhoff, P. \& Geiger, H. H. Quantitative trait loci for early plant vigour of maize grown in 552 chilly environments. Theor. Appl. Genet. 114, 1059-70 (2007).

553

554

555

556

557

558

559

560

561

Reimer, R. Response of maize (Zea mays L.) seedlings to low and high temperature : association mapping of root growth and photosynthesis-related traits presented by no. 18807. $(\mathrm{PhD}$ thesis, Swiss Federal Institute of Technology, Zurich, Switzerland, 2008).

Keane, G. P. Agronomic factors affecting the yield and quality of forage maize in Ireland: Effect of sowing date and plastic film treatment. Grass Forage Sci. 57, 3-10 (2002).

Levitt, J. Chilling, Freezing, and high Temperature Stresses in Responses of Plants to environmental Stress. $2^{\text {nd }}$ Edn. New York, NY, USA: (Academic Press, 1980).

Liedgens, M. \& Richner, W. Minirhizotron observations of the spatial distribution of the maize root system. Agron. J. 93, 1097-1104 (2001). 
562 Ludwig, A. \& Tenhaken, R. (2001) Suppression of the ribosomal L2 gene reveals a novel

563 mechanism for stress adaptation in soybean. Planta 212, 792-798.

564 Marocco, A., Lorenzoni, C. \& Fracheboud, Y. Chilling stress in maize. Maydica 50, 571-80 $565 \quad$ (2005).

566 Miura, K. \& Furumoto, T. Cold signalling and cold response in plants. Int. J. Mol. Sci. 14, 5312$5675337(2013)$.

568 Nguyen, H. T., Leipner, J., Stamp, P. \& Guerra-Peraza, O. Low temperature stress in maize (Zea 569 mays L.) induces genes involved in photosynthesis and signal transduction as studied by 570 Suppression Subtractive Hybridization. Plant Phys. Biochem. 47, 116-122 (2009).

571 Nie, G.Y., Long, S. P. \& Baker N. R. The effects of development at dub-optmial growth 572 temperatures on photosynthetic capacity and susceptibility to chilling-dependent 573 photoinhibition in Zea mays. Physiol. Plant. 85, 554-60 (1992).

574 Puranik, S., Sahu, P.P., Srivastava, P.S., \& Prasad, M. (2012) NAC proteins: regulation and role 575 in stress tolerance. Trends Plant Sci. 17, 369-381.

576 Revilla P., Rodriguez V. M., Ordas A., Rincent R., Charcosset A., Giauffret C., Melchinger A. 577 E., Schon C.C., Bauer E., Altmann T., Brunel D., Gonzalez J. M., Campo L., Ouzunova M., 578 Alvarez A., de Galarreta J. I. R., Laborde J., \& Malvar R. A. Association mapping for cold 579 tolerance in two large maize inbred panels. BMC Plant. Biol. 16: 127 (2016). 
580 Ruta, N., Liedgens, M., Fracheboud, Y., Stamp, P. \& Hund, A. QTLs for the elongation of axile 581 and lateral roots of maize in response to low water potential. Theor. Appl. Genet. 120: 621582 $31(2010)$.

583

584

585

586

587

588

589

590

591

592

593

594

595

596

597

Sanguineti, M. C., Giuliani, M., Govi, G. \& Tuberosa, R. Root and shoot traits of maize inbred lines grown in the field and in hydroponic culture and their relationships with root lodging. Maydica 43, 211-16 (1998).

Seifert, F., Thiemann, A, Pospisil, H. \& Scholten, S. Re-annotation of the maize oligonucleotide array. Maydica 57, 49-55 (2012).

Sobkowiak, A., M., Jończyk, E., Jarochowska, P., Biecek, J., Trzcinska-Danielewicz, J., Leipner, J., FronkJ. \& Sowiński P. Genome-wide transcriptomic analysis of response to low temperature reveals candidate genes determining divergent cold-sensitivity of maize inbred lines. Plant Mol. Biol. 85, 317-331 (2014).

Stamp, P. Yield performance of tropical highland maize and its top crosses with middle European hybrids. Z. Acker Pflanzenbau 153, 366-72 (1984).

Theocharis, A., Clément, C. \& Barka E. A. Physiological and molecular changes in plants grown at low temperatures. Planta 235, 1091-1105 (2012).

Tollenaar, M. \& Lee, E. A. Yield Potential, Yield stability and stress tolerance in maize. Field Crops Res. 75, 161-69 (2002). 
598 Untergasser, A., Nijveen, H., Rao, X., Bisseling, T., Geurts, R. \& Leunissen, J. A. Primer3Plus, 599 an enhanced web interface to Primer3. Nucleic Acids Res. 35, W71-4 (2007).

600 Vandesompele, J., De Preter, K., Pattyn, F., Poppe, B., Van Roy, N., De Paepe, A. \& Speleman, 601 F. Accurate normalization of real-time quantitative RT-PCR data by geometric averaging of 602 multiple internal control genes. Genome Biol. 3 (2002).

603 Van Loon, L. C., Rep, M. \& Pieterse, C. M. J. Significance of inducible defense-related proteins 604 in infected plants. Ann. Rev. Phytopatol. 44, 135-62 (2006).

605 